\title{
Hacia una visión ecológica de la educación especial para zonas rurales e indígenas
}

\author{
Guadalupe Acle Tomasini ${ }^{1}$, \\ Ma. del Pilar Roque Hernández, \\ y Eduardo Contreras Ramírez ${ }^{2}$
}

${ }^{1}$ División de Estudios de Posgrado, Facultad de Estudios Superiores Zaragoza, Universidad Nacional Autónoma de México, México/Distrito Federal ${ }^{2}$ Licenciatura en Psicología, Facultad de Estudios Superiores Zaragoza, Universidad Nacional Autónoma de México, México/Distrito Federal

\section{México}




\section{Resumen}

Introducción. Los modelos ecológicos que abordan el estudio de fenómenos educativos, plantean que los alumnos están implicados en múltiples ambientes en los que juegan diversos papeles; en cada uno se espera muestren ciertos comportamientos; en ocasiones esto puede crear conflictos que pueden deberse a la discrepancia existente entre la habilidad del individuo para llenar los requisitos del ambiente o porque éste no satisface sus necesidades. Desde esta perspectiva, este estudio buscó determinar la manera en que se manifiestan las necesidades educativas especiales en niños indígenas al inicio de su aprendizaje escolar así como analizar la relación que éstas guardan con las distintas fuerzas ambientales que rodean a los menores.

Método. El uso de metodología cualitativa, consistió en el empleo de técnicas etnográficas, que permitieron registrar datos de los contextos escolar, familiar y cultural. La metodología cuantitativa se utilizó en la aplicación del instrumento psicoeducativo a 96 niños otomíes, 50 niños y 46 niñas, cuyos resultados se correlacionaron con diferentes variables tales como ocupación de los padres, el que éstos supieran leer, género y edad de los menores.

Resultados. Se caracterizan las discrepancias existentes entre las habilidades de los niños adquiridas en el hogar y las requeridas en la escuela. Los datos, tanto cuantitativos como cualitativos recabados, permiten documentar las dificultades que niños otomíes presentan al ingresar a la educación primaria; diferencias significativas en el desempeño de las habilidades se encuentran entre quienes repiten el grado y cursan el preescolar y los que no. El medio cultural y familiar delimita las expectativas educativas así como la permanencia en la escuela.

Discusión. Utilizar un enfoque ecológico para detectar los requerimientos educativos especiales de niños de zonas rurales e indígenas, e intervenir en consecuencia, muestra la necesidad de incluir otras variables de análisis, que en su interacción con el menor permitan una comprehensión mayor de este objeto particular de estudio. Es claro que la tarea no es fácil, pero tampoco se puede seguir centrando en el niño la responsabilidad y mantenimiento de este tipo de dificultades, en particular, en estas poblaciones, en las que la inadecuada aplicación e interpretación de tests, puede añadir un elemento más de marginación: el de la discapacidad.

Palabras clave: Necesidades especiales, ecología educativa, indígenas, evaluación. 


\section{Introducción}

Los enfoques ecológicos abocados a la explicación del aprendizaje escolar, enfatizan la manera en que el ambiente ejerce sobre el niño una acción continua y permanente, por lo que es necesario considerar su influencia a través de diversas personas, situaciones y lugares. Aún cuando se ha incrementado la investigación utilizando este enfoque, existe poca evidencia de cómo conceptuar los problemas con objeto de llevar a cabo una intervención desde esta perspectiva. Para Swartz y Martin (1997) la utilización de este enfoque debe lograr cambios en diferentes ecosistemas, sobre todo, por la multiplicidad y diversidad de ambientes en que el individuo funciona.

Las comunidades, familias, escuelas e individuos forman parte de las redes ecológicas al interior de los ecosistemas, por lo tanto, una intervención apropiada se fundamentará en el adecuado entendimiento de estas redes. El niño está inmerso en una compleja interacción con diversas fuerzas ambientales. Para Báez de la Fé (1988) las variables dinámicas consideradas dentro de esta postura son: el alumno, el aula, la infraestructura, la familia y el entorno. En el campo de la educación especial, Kauffman (1995) indica que los principios ecológicos son importantes, pues, con las políticas actuales de integración educativa se afirma la necesidad de construir un sistema social general que sea hospitalario e incluya una variedad de ambientes, dirigidos a la diversidad de las características de la población.

Las características tanto internas como externas de la interacción entre el individuo y el medio determinan los comportamientos de la persona, de aquí que, se enfatice desde esta perspectiva, el estudio de la interacción de los sistemas en los que el individuo funciona (Swartz y Martin, 1997). Las orientaciones ecológicas actuales buscan: (a) describir los contextos en los que está inmerso el individuo; (b) conceptuar al ambiente ecológico desde múltiples niveles de análisis; (c) reconocer las diferencias entre los diversos ambientes; (d) aceptar que la conducta del individuo es transaccional y no puede ser entendida fuera del contexto en que ocurre; y, (e) la creencia de que las intervenciones deben construirse para ecologías específicas.

La cultura es el medio a través del cual el individuo se adapta a las demandas del ambiente, los enfoques ecológicos asumen el concepto de homeostasis; en este sentido, la cultura 
es la que provee los elementos para el ajuste y la adaptación, sean éstos biológicos, psicológicos o culturales, pueden o no, formar parte del repertorio conductual del sujeto, puede incluso haber disparidad entre el individuo y el ambiente. Para Aguirre Beltrán (1992), el análisis de la educación debe comprenderse en el contexto en que se lleva a cabo, para él la acción integral en la educación indígena, implica conjugar campos de actividad que normalmente se instrumentan de manera separada tales como: la educación escolarizada, la salubridad, la economía y la agricultura. En términos llamados "ecológicos", el autor enfatizó la interacción del hombre y su hábitat en una unidad geográfico-social más o menos homogénea, que denominó región. Cuando esta región es intercultural, la acción integral propuesta en la educación debe tomar en cuenta a los grupos humanos en contacto.

Los modelos ecológicos que abordan el estudio de fenómenos educativos, plantean que los alumnos están implicados en múltiples ambientes en los que juegan diversos roles, en cada uno de ellos, se espera muestren ciertos comportamientos, lo que en ocasiones crea conflictos que pueden deberse a la discrepancia existente entre la habilidad del individuo para llenar los requisitos del ambiente, o bien, porque éste no satisface las necesidades del sujeto (Swartz \& Martin, 1997). Un aspecto clave de estos modelos, es que modifican el planteamiento que afronta el fenómeno educativo centrándose sólo en la persona al proponer el estudio de la relación entre ésta y los diversos elementos de su medio (Acle, 2003). El enfoque ecológico es considerado como un marco para guiar la manera en que se piensa acerca de la escuela y el aprendizaje (Acle, 2000; Adelman y Taylor, 1994).

Lo anteriormente citado, atañe igualmente a los procedimientos de evaluación e intervención; autores como Rosenberg (1987), Sugai y Maheady (1988) recomiendan que la valoración que se realice con personas de culturas diferentes, incluya: la adquisición de habilidades básicas, el contenido de las materias, la interacción sistemática del grupo y de la instrucción, así como las oportunidades de aprendizaje vividas. La evaluación debe basarse en los fundamentos de la perspectiva ecológica: ofrecer una visión comprehensiva del problema, no descartar formas tradicionales de evaluación, al contrario, integrarlas con objeto de que puedan conocerse todos los sistemas que interactúan; y, proponer como meta principal el estudio de la relación entre las expectativas entre el ambiente y los individuos, para identificar las conductas, escenarios y condiciones en las que se tendrá que intervenir (Adelman y Taylor, 1994, Swartz y Martín, 1997). 
La utilización de esta perspectiva, en el estudio de aspectos educativos referidos a poblaciones indígenas, adquiere relevancia si se considera que la población indígena de México se conforma de 12 millones setecientas mil personas. Sólo el $41 \%$ de niños indígenas de cinco años asiste a la escuela y de los de seis a 14 años el 87.2\% lo hace. De la población de quince años y más sólo el $67.5 \%$ es alfabeta; en este rango se reporta además que, el $25.8 \%$ no ha recibido instrucción, el $27.2 \%$ tiene estudios de primaria incompleta, primaria completa el 18.7\% y con estudios posteriores a la primaria el 28.2\% (Serrano, Embriz y Hernández, 2002). A estos porcentajes, de por sí significativos, se añaden otras consideraciones relativas a las enormes diferencias geográficas, culturales y económicas que existen en un país como México, en el que se concentran mayores beneficios en las grandes urbes y se sigue dejando marginados de la educación a muchos mexicanos, de entre ellos a las poblaciones indígenas.

La población rural e indígena comparte con muchos sectores populares una condición de pobreza y de marginación social. La desigualdad social y económica de este tipo de comunidades, son factores que no únicamente determinan el acceso diferencial al sistema escolar sino también los distintos mecanismos de incorporación a los diversos niveles del sistema educativo. En este sentido, la incorporación de servicios de educación especial es reciente, data del sexenio 1988-1994, cuando aparece estipulado por primera vez en el Programa de Modernización Educativa 1989-1994 el objetivo de brindar atención en las zonas rurales e indígenas, de lo cual surgió un cuestionamiento básico: ¿Bajo qué concepto de educación especial iba a trabajarse?. Para los propósitos de la educación especial, una diferencia estará constituida por una marcada discrepancia, superior o inferior, en la ejecución de cualquier tipo de tarea, de un individuo en relación con la de la mayoría (Kirk y Gallagher, 1989). Pero: ¿Bajo qué circunstancias la ejecución de los individuos puede ser considerada como superior o inferior? En un país como México con tantas diferencias económicas y socioculturales, tanto en la ciudad como en el campo, ¿qué es lo que se va a considerar como desviado de la norma?

Dicha propuesta llevó a reflexionar acerca de cómo se establecería la frontera entre la educación especial y la regular en zonas rurales e indígenas. Reflexión que se destacó cuando, al proponer un modelo de educación especial para el medio indígena, Izquierdo, Fernández y Nieto (1992) enfatizaron la necesidad de caracterizar la educación especial indígena considerando sus principios, ámbitos y cultura. Para dar seguimiento a lo establecido en dicho programa, la Dirección General de Educación Especial (D.G.E.E.) diseñó un proyecto: "Detec- 
ción de la Demanda de Educación Especial en zonas rurales e indígenas" (s/f), en el que se reconocían las desigualdades educativas de este tipo de zonas así como las variaciones presentes en las distintas comunidades y grupos étnicos.

Para tal efecto, Paéz y Trejo (1994) elaboraron una "Guía de Observación para el Docente", cuestionario compuesto de 80 reactivos dicotómicos que fue contestado por los maestros de las zonas rurales elegidas para el estudio. Esta guía, se enfocó principalmente a determinar la presencia de cuatro categorías: (a) deficiencia mental; (b) problemas auditivos; (c) problemas neuromotores; y, (d) problemas visuales. De los resultados obtenidos, las autoras indicaron que en 432 niños de la población total de la muestra $(n=2260)$, se podían diferenciar dos grupos: uno de 174 menores (40.3\%) que presentaban algún tipo de déficit correspondiente a las categorías definidas en la guía y, otro de 258 niños (59.7\%) que manifestaban problemas de aprendizaje y/o conducta. Datos significativos, pues, indican que el $19.11 \%$ de la muestra estudiada se ubicó con requerimientos de educación especial, porcentaje que rebasa en mucho el 10\% estipulado por la Organización Mundial de la Salud para la población total de un país.

A partir de estos antecedentes, desde 1995 se ha llevado a cabo un proyecto intitulado "Diseño de un Modelo Ecológico de Evaluación-Intervención de Necesidades Educativas Especiales en una Zona Rural e Indígena” el cual parte de los supuestos básicos del enfoque ecológico antes mencionados además de enfatizar que:

(1) La escuela constituye un hábitat creado por el hombre, ubicado de manera particular en un ecosistema conformado por aspectos políticos, sociales, jurídicos, económicos, culturales y educativos.

(2) La noción de interrelación dinámica entre los distintos elementos de un ecosistema es un aspecto clave, pues, un sistema refleja un conjunto de elementos que están entrelazados y que por consiguiente interactúan entre sí, cuando uno de ellos se mueve, se modifica todo el conjunto, todos los elementos tienen igual peso, ya que, el todo es mayor que la suma de las partes (Acle, 2003).

El propósito del proyecto fue precisamente, mostrar la manera en que la interacción entre los diversos actores -niños, padres, maestros, administración escolar- y elementos cultura, salud, bilingüismo, política educativa, autoconcepto, contenidos y materiales de aprendizaje- permite documentar la aparición, mantenimiento y erradicación de las necesida- 
des educativas especiales (NEE) en niños otomíes. Se ha trabajado en el municipio de Temoaya, estado de México, cuya población es otomí, la zona es bilingüe, el 97\% habla una lengua indígena, siendo el otomí la primera lengua. De su población de más de 15 años, el $20.4 \%$ no tiene algún tipo de instrucción, el 35\% tiene estudios incompletos de primaria, primaria completa el 21.2\% y, sólo el 23.2\% estudió algo posteriormente (Serrano, et al., 2002).

Una tarea primordial dentro del proyecto ha sido la de detectar al inicio del año escolar, en una escuela monolingüe español, a niños otomíes de primer grado con dificultades de aprendizaje, así como, analizar las condiciones externas bajo las cuales la falta de rendimiento se presentaba, considerando que, sólo en el primer grado, los alumnos reportados por los maestros con dificultad para aprender ha sido desde entonces de un $20 \%$ en promedio. En el primer estudio exploratorio, Acle y Roque (1994) encontraron la presencia de deficiencias en la realización de tareas de lenguaje expresivo y de lenguaje escrito, tales como dictado y copia. No obstante, en cuanto a los instrumentos aplicados, pese a que aportaron información para caracterizar las dificultades encontradas, mostraron su falta de adecuación y la severa penalización que niños pertenecientes a grupos rurales indígenas pueden sufrir. A partir de estos hallazgos se elaboró un instrumento específico orientado a detectar dificultades en las áreas visomotora y auditivo -verbal, precurrentes básicas para el logro del aprendizaje escolar, se tuvo relación constante con el maestro a fin de que en las tareas solicitadas a los niños se reflejaran las actividades enseñadas en clase (Acle, Roque y Contreras, 1996).

Hallazgos significativos de este estudio fueron: (1) La heterogeneidad en cuanto a edades de los niños, sólo el $48 \%$ contaba con los seis años, edad reglamentaria en México para ingresar al primer grado de primaria; el porcentaje restante se ubicaba entre los 7 y 10 años. (2) Sólo el 41\% había cursado algún grado de preescolar. (3) En el área verbal, resaltó el que los grupos de mayor edad fueran menos capaces que los pequeños de agrupar colores teniendo los estímulos presentes. En cuanto a actividades de representación, se observó que los pequeños no eran capaces aún de formar campos semánticos sin los objetos. (4) Los niños otomíes participan en actividades domésticas y laborales como parte de la organización social que la misma familia tiene. De aquí la necesidad de considerar la familia-entorno, como un elemento indispensable para el entendimiento del interés y participación tanto del niño como de sus padres, en su propio aprendizaje. (5) Al interior del aula, y en lo que concierne a la interacción maestro-alumno, no se observó el establecimiento de una relación auténtica y de confianza, ni la promoción de la autorregulación y la auto-organización de los niños, ya que el 
maestro sigue utilizando técnicas pasivas de enseñanza, establecidas en el programa oficial que él debe aplicar, sin considerar patrones culturales de la vida cotidiana de estos niños indígenas (Acle, Roque y Contreras, 1996).

El propósito del presente estudio fue determinar la manera en que se manifiestan las necesidades educativas especiales en niños indígenas que ingresan al primer grado de educación primaria, así como analizar la forma en que, la presencia de éstas, se vincula con las características y demandas de las distintas fuerzas ambientales que rodean al menor, en particular las los medios familiar, cultural y escolar.

\section{Método}

\section{Participantes}

La selección de la muestra fue del tipo no probabilístico intencional. Los participantes fueron 96 niños otomíes, 50 niños y 46 niñas, cuyas edades se ubicaron entre los 5 años 7 meses a los 8 años 10 meses ( $\mathrm{X}=6$ años 5 meses), el 52\% de la muestra había cursado el preescolar y, el 12\% eran repetidores. Datos relevantes respecto a sus padres fueron: sólo el 76\% de manifestó saber leer, el 25\% eran comerciantes, el 65.6\% se dedicaba al hogar, el 3\% trabajaba como empleada doméstica y sólo el 1\% reportó haber realizado una carrera profesional, la de maestro de educación primaria. El promedio de escolarización de las madres, fue el segundo grado de educación primaria.

\section{Instrumento}

El instrumento de detección elaborado ex profeso estuvo conformado de dos aspectos principales: el área visomotora y la auditivo-vocal. Para la primera, se utilizaron pruebas de lápiz y papel con las que se evaluaron las habilidades de: discriminación de letras, integración visomotora y dibujo de la figura humana. En la segunda, se consideraron las habilidades de: conocimiento del color, el manejo de campo semántico -animales, plantas, frutas-, memoria auditiva inmediata en la repetición de dígitos y en la repetición de frases. La calificación de cada uno de los reactivos fue de 0 a 10 . En la elección de las tareas se consideraron por un lado aspectos de madurez visomotora esperados a esa edad, así como las actividades escolares que habían sido trabajadas con el maestro. Se llevaron a cabo constantes entrevistas con los 
maestros que impartían el primer grado, algunos padres y directivos de la escuela, además del registro de observaciones del contexto cultural.

\section{Contexto y escenario}

Temoaya es un municipio conformado por 38 localidades indígenas, se ubica a 20 kms. de Toluca y a 80 del Distrito Federal. Como se señaló, la zona es bilingüe; el 97\% habla una lengua indígena, siendo el otomí la primera lengua (Serrano et al., 2002). La población es campesina con una fuerte migración, en particular masculina. El número de escuelas registradas para 1995 era de 121, de las cuales 54 correspondían al preescolar, 48 a la primaria, 16 al nivel de secundaria, una preparatoria, un Centro de Capacitación en Artes y Oficios (CECAO) y un Colegio Nacional de Educación Profesional Técnica (CONALEP). La relación del número de escuelas de primaria con las de nivel medio y medio superior sigue siendo piramidal y, no es proporcional, si se considera que los índices demográficos del municipio, muestran que el 59\% de la población total corresponde a personas menores de 19 años (Acle y Roque, 1994; Acle, 2003).

En este contexto, el escenario en el que se ha trabajado es una escuela monolingüe en español ubicada en una de las localidades indígenas de dicho municipio. Una característica importante de este medio, es que la población escolar que asiste es indígena a diferencia de los docentes y administradores escolares. Cabe mencionar que es una escuela con prestigio dentro de la comunidad y a ella asisten en promedio 510 menores, se imparte el programa oficial establecido por el Ministerio de Educación para todas las escuelas del país. En términos generales, se observa que la relación maestro/alumnos en el ciclo escolar 1998-99 fue de uno por 36, lo cual, como señalan los docentes, beneficia el proceso de enseñanza aprendizaje.

\section{Procedimiento}

Es importante tener presente que, tanto la metodología cuantitativa como la cualitativa, han considerado a la educación especial como su objeto de estudio, empleando procedimientos sistemáticos al realizar investigaciones (Roque, 2001). En este sentido, la metodología cuantitativa ha sido básica para sistematizar el análisis de los resultados derivados de la aplicación de instrumentos y la comparación entre variables. La metodología cualitativa utili- 
zada ha sido fundamentalmente etnográfica, apoyada en instrumentos como: registros de observación, guías de entrevista y, obtención de información de diversas fuentes.

Se asistió a la escuela primaria de la localidad, se solicitó autorización al director para aplicar el instrumento, éste se aplicó a los niños que recién habían ingresado al $1^{\mathrm{o}}$ año y que estaban ubicados en tres grupos. La aplicación de la parte visomotora fue grupal y se hizo en una sesión de hora y media aproximadamente. La maestra de cada grupo estuvo presente y colaboró en la aplicación. En lo que se refiere a la parte auditivo-verbal, ésta se aplicó de manera individual y se ocupó aproximadamente 40 minutos en cada niño. El instrumento se calificó en cada aspecto con valores de uno a diez. Se tuvo contacto constantemente con el maestro para tener su opinión respecto a: (1) los menores que a su juicio tenían dificultad y (2) los que habían sido detectados por nosotros. Se llevaron a cabo además, observaciones de su interacción con los niños dentro y fuera del aula. Así como se llevó un registro de la dinámica general de la escuela y de los censos escolares.

Se llevó a cabo un análisis descriptivo que permitió observar la ejecución de los niños en cada uno de los reactivos empleándose la prueba t de Student para muestras independientes con objeto de evaluar sí existían diferencias en la ejecución de las tareas con relación a la asistencia al preescolar y el ser reptidor. Para valorar la relación entre la ejecución en las áreas y las variables: género, edad, conocimiento de la lectura de los padres, ocupación y edad de las madres se utilizó la correlación de Spearman.

La información obtenida de las entrevistas y el registro de las observaciones se analizó cualitativamente, organizándose en las siguientes categorías de análisis: relación maestroniño, maestro-familia y familia-entorno.

\section{Resultados}

Un primer aspecto que conviene comentar en referencia a la edad de los niños; es que aún cuando la media del grupo corresponde a la edad esperada para cursar el primer grado: 6 años 5 meses, se observó que el $11.5 \%$ de la muestra era mayor de 8 años, porcentaje que a su vez correspondía al número de menores que se encontraba cursando por segunda vez el primer grado de educación primaria. Al igual que en el estudio llevado a cabo en 1996 por Acle, Roque y Contreras, se advirtió la heteregoneidad en las edades de los menores, lo cual se rela- 
ciona con la importancia que tiene el que los niños indígenas asistan a la escuela, por lo que se flexibilizan los requisitos de ingreso a diferencia de las escuelas de medios urbanos en las que se es estricto en el cumplimiento de la edad reglamentaria para el ingreso a primaria.

La ejecución del grupo respecto a las áreas evaluadas se muestra en la Tabla 1. Puede observarse un promedio significativamente bajo en las tareas de reconocimiento de figuras geométricas y de memoria auditiva inmediata. No obstante, cabe mencionar que al llevar a cabo el análisis de frecuencias para cada una de las tareas se advierte que, un porcentaje significativo de niños se ubica en el grupo inferior de ejecución en: discriminación letras, integración visomotora, identificación de colores y de las figuras geométricas.

Tabla 1. Análisis descriptivo de las calificaciones obtenidas en las habilidades evaluadas.

\begin{tabular}{|c|c|c|c|c|c|}
\hline $\begin{array}{l}\text { Área Visomoto- } \\
\text { ra }\end{array}$ & $\begin{array}{c}\text { Calificación } \\
\text { Mínima }\end{array}$ & $\begin{array}{c}\text { Calificación } \\
\text { Máxima }\end{array}$ & Media & $\begin{array}{l}\text { Desviación } \\
\text { estándar }\end{array}$ & $\begin{array}{c}\text { \% de } n \text { en } \\
\text { grupo inferior }\end{array}$ \\
\hline $\begin{array}{l}\text { Discriminación } \\
\text { de letras }\end{array}$ & 0 & 10 & 6.9 & 1.9 & 46.3 \\
\hline $\begin{array}{l}\text { Integración } \mathrm{Vi-} \\
\text { somotora }\end{array}$ & 2.8 & 10 & 7.7 & 1.6 & 22.9 \\
\hline Figura Humana & 0 & 10 & 10 & 0 & 0 \\
\hline $\begin{array}{l}\text { Área auditivo- } \\
\text { verbal }\end{array}$ & & & & & \\
\hline Color & 0 & 10 & 7.7 & 3.6 & 36.8 \\
\hline $\begin{array}{l}\text { Figuras Geomé- } \\
\text { tricas }\end{array}$ & 0 & 10 & 2.9 & 3.3 & 89.5 \\
\hline $\begin{array}{l}\text { Campo semánti- } \\
\text { co }\end{array}$ & 0 & 10 & 9.8 & 1.2 & 5.3 \\
\hline $\begin{array}{l}\text { Memoria Auditi- } \\
\text { va Inmediata }\end{array}$ & 0 & 10 & 5 & 2.2 & 72.3 \\
\hline $\begin{array}{l}\text { Repetición de } \\
\text { Frases }\end{array}$ & 5 & 10 & 9.8 & .72 & 2.1 \\
\hline
\end{tabular}


Aún cuando todos los menores pudieron dibujar la figura humana, 33\% de ellos lo hizo de forma demasiado simple. Estos resultados son significativos e indicativos de la posible presencia de NEE, se vinculan, por un lado, con la limitada asistencia al nivel preescolar, pues de la muestra, sólo asistió el $52 \%$ y, por otro, con las diferencias culturales entre casa y escuela. Ahora bien, no obstante que el 48\% restante cursó preescolar, muchos de los menores sólo lo hacen el año previo a ingresar a la primaria; las mamás señalaron que no los enviaban antes por razones económicas. Por su parte, los docentes señalaron que un año de preescolar no es suficiente.

Para valorar sí habían diferencias entre las variables de asistencia al preescolar y el ser repetidor con la ejecución de las tareas solicitadas, se aplicó la prueba t de Student para muestras independientes; los resultados mostraron que los niños que asistieron al preescolar en comparación con los que no lo cursaron, realizaron mejor algunas de las tareas como la de integración visomotora $(\mathrm{t}=2.310, \mathrm{p}=<.02)$ y la de nombrar correctamente las figuras geométricas básicas $(\mathrm{t}=4.027, \mathrm{p}=<.00)$, lo cual habla a favor de la asistencia a dicho nivel. Así mismo los niños repetidores tuvieron una mejor ejecución en integración visomotora $(\mathrm{t}=$ $2.330, \mathrm{p}=<.04)$ y en discriminación de letras $(\mathrm{t}=2.443, \mathrm{p}=<.03)$ lo que pone de manifiesto el efecto de la escolarización recibida.

Para determinar si existía un grado de asociación entre las variables: género, edad de los menores, asistencia al preescolar, el que los padres sepan leer, la ocupación que desempeñan y la edad de las madres con la ejecución en las tareas evaluadas, se realizó un análisis de correlación (Tabla 2.). Éste mostró relaciones positivas significativas entre la ocupación que desempeñan las madres con el desempeño en discriminación de letras $(r=.208)$ y en memoria auditiva inmediata $(r=.232)$, lo cual puede relacionarse con la manera en que ellas indican ayudarles a hacer sus tareas. Así como, entre la edad de los menores y su desempeño en integración visomotora $(\mathrm{r}=.270)$ y el manejo de campos semánticos $(\mathrm{r}=.224)$ lo que tiene que ver con aspectos de su propio desarrollo.

No obstante, se apreciaron correlaciones negativas significativas entre el hecho de que los padres sepan leer con el resultado obtenido por los niños en las tareas de integración vi- 
somotora $(\mathrm{r}=-.228)$ y el conocimiento de los colores primarios $(\mathrm{r}=-.268)$, así como en la asistencia al preescolar con el desempeño en integración visomotora $(r=-.228)$, conocimiento de colores primarios $(r=-.291)$ y el de las figuras geométricas $(r=-.399)$, lo cual indica que no existe relación entre estos factores externos y el desempeño de los niños en estas áreas básicas, baste recordar que el $24 \%$ de padres de los menores no sabían leer y el $48 \%$ de los menores no cursó preescolar, de hecho, en relación con el útlimo aspecto, se había encontrado un mejor desempeño entre quienes sí habían asistido a este nivel educativo.

Tabla 2. Resultados de las correlaciones entre lasvariables establecidas.

\begin{tabular}{|c|c|c|c|c|c|c|}
\hline $\begin{array}{l}\text { Área Visomo- } \\
\text { tora }\end{array}$ & Género & Edad & $\begin{array}{l}\text { Asistencia } \\
\text { a Preesco- } \\
\text { lar }\end{array}$ & Saber leer & $\begin{array}{l}\text { Ocupación } \\
\text { de los pa- } \\
\text { dres }\end{array}$ & $\begin{array}{ll}\text { Edad de } \\
\text { las ma- } \\
\text { dres }\end{array}$ \\
\hline $\begin{array}{l}\text { Discriminación } \\
\text { de letras }\end{array}$ & .071 & .161 & -.037 & -.099 & $.208 *$ & .171 \\
\hline $\begin{array}{l}\text { Integración Vi- } \\
\text { somotora }\end{array}$ & -.127 & $.270 * *$ & $-.228 *$ & $-.228 *$ & .120 & .004 \\
\hline
\end{tabular}

Área auditivo-

verbal

\begin{tabular}{lllllll}
\hline Color & -.049 & .153 & $-.291^{* *}$ & $-.268^{*}$ & .151 & -.090 \\
$\begin{array}{l}\text { Figuras Geomé- } \\
\text { tricas }\end{array}$ & .046 & .020 & $-.399^{* *}$ & -.111 & .049 & .013 \\
Campo semán- & -.057 & $.224^{*}$ & -.134 & -.085 & -.083 & .059 \\
tico & & & & & & \\
Memoria Audi- & .108 & .029 & -.117 & -.038 & $.232^{*}$ & .037 \\
tiva Inmediata & & & & & \\
Repetición de & .142 & -.167 & -.017 & .081 & -.054 & .040 \\
Frases & & & & &
\end{tabular}
$* * \mathrm{p}=<.01, * \mathrm{p}=<.05$

Datos relevantes de las observaciones y entrevistas indicaron que, al interior del aula y, en lo que concierne específicamente a la interacción maestro-alumno se apreció, por ejemplo, 
a docentes de primer grado que no promovían ni la participación ni la autorregulación de los menores, quienes constantemente se desplazaban de su lugar y se acercaban al maestro con objeto de pedir su aprobación y enseñarle lo que habían hecho. Cuando se explicaba algún tema, los niños, al no entender lo que se les decía, se copiaban y ayudaban unos a otros. Ser exigente se traducía en alzarles la voz, era lo que iba a ayudar para que los niños lograran su autocontrol e independencia. En lo que concierne al trabajo académico, existe, por el contrario, rigidez en cuanto al tiempo en que los niños deben adquirir el aprendizaje; en el curriculum formal se determinan los límites: todos los niños deben aprender a leer y a escribir en tiempos iguales con objeto de dar cumplimiento a los programas establecidos por el Ministerio de Educación.

En este sentido, algunos docentes muestran una preocupación real porque los niños aprendan, otros, se interesan más por el cumplimiento de los requerimientos administrativos. La percepción que los maestros de esta primaria rural manifiestan, respecto de la participación de los padres en la educación escolarizada de sus hijos, así como de las deficiencias de los niños, se derivan, en algunos casos, de actitudes negativas hacia ciertas características culturales de esta población. En el caso de los maestros que se preocupan por el aprendizaje de los niños, se pudo advertir el propio reconocimiento de la falta de preparación para enseñar a niños con dificultades de aprendizaje; en general se sienten solos, saben que deben sacar a los niños adelante pues, no tener reprobados es importante, pero también reconocen que no es fácil porque "los padres no colaboran", "son los padres los que forman los hábitos en los niños para mantener la atención y estudiar fuera de la escuela".

En contraposición, es relevante indicar que, para los padres entrevistados, el valor de la escuela es totalmente funcional; se relaciona con la importancia de aprender a leer, escribir, hablar y entender el español, para que en el futuro sus hijos puedan integrarse al comercio y encontrar trabajo. Por otro lado, los padres influenciados por su condición socioeconómica y cultural, asumen que sólo el profesor sabe, así, aún cuando tengan dudas no se atreven a preguntar. A su manera, ellos ayudan a sus hijos a hacer sus tareas para que: "...entienda y aprenda, lo haga bien y rápido, no lo castiguen o regañen y a mi no me reclamen, aprovechando el dinero que se gasta en él...", "...aunque no sé leer ni escribir, lo pongo a trabajar, a que llene los cuadernos, lo vigilo, le exijo, le pego, lo apuro o lo regaño...". Una queja de las 
madres entrevistadas fue que los maestros nunca les explican qué significan las calificaciones o las correcciones que hacen en los cuadernos, sólo les dicen que los niños están mal y que tienen que hacer qué estudien, pero, no les indican cómo hacerlo, lo que dado su propio nivel educativo y lingüístico se les dificulta.

En lo referente al vínculo que los maestros guardan con los padres, es interesante observar que muchos de ellos consideran que es mejor que los padres no intervengan en la educación, algunos señalaron porque: "...no tiene utilidad su participación, pues son incapaces de aportar y ayudar en algo, todos los problemas que surgen son por ellos", "...por los pocos conocimientos que tienen y por que tienen otras prioridades que son las que les ayudan a subsistir". Estos comentarios, muestran las diferencias acerca de la relevancia cultural del aprendizaje escolar; pareciera que ésta opera, entre maestros y padres, de forma paralela. A lo que se añade el hecho, de que no se ha logrado el establecimiento de mecanismos específicos de colaboración maestro-padres, en específico en los aspectos académicos pues existe para otras actividades, como es la participación en la organización de diversos eventos: desayunos escolares, fiestas o ceremonias.

\section{Discusión}

El surgimiento de los enfoques ecológicos en la explicación de los problemas de aprendizaje es relativamente reciente y tiene su repercusión en la medida en que se aborda la influencia que tiene el medio sobre su presencia, mantenimiento o erradicación. Participar en un contexto cultural diferente al propio, lleva a la necesidad de conceptuar nuevos planteamientos y métodos diferentes de evaluación e intervención. La relevancia que esto adquiere en la caracterización de los problemas de aprendizaje y las necesidades educativas especiales en poblaciones indígenas, es innegable, sobre todo, si se consideran los bajos índices educativos en estas poblaciones y, no sólo en el caso de México. Los datos obtenidos en el presente estudio dan muestra de los diferentes elementos que interactúan para promover o no el aprendizaje escolar desde que los niños indígenas lo inician.

El instrumento aplicado a los menores da cuenta de lo señalado por Swartz y Martín (1997) en relación con la discrepancia que existe entre las habilidades que presentan los menores y las que son requeridas para el logro de las ejecuciones escolares. Lo cual a su vez, 
tiene que ver con las diferencias lingüísticas y culturales entre el hogar y la escuela, pues, es innegable que los niños llegan a la escuela con conocimientos previos, sólo que, éstos no son los que la escuela demanda; por ejemplo, en su habilidad de coordinación motriz fina son capaces de desgranar el maíz pero no en tareas de lápiz y papel. De hecho, al considerar los bajos ingresos familiares así como los del nivel de escolarización de los padres, poco acceso tienen los menores a materiales escritos hasta que ingresan a la educación primaria, a partir de los libros de texto gratuito que se les entregan.

Estas discrepancias en las habilidades requeridas por la escuela se reflejaron en los siguientes resultados: (a) $46.3 \%$ de la muestra tuvo dificultad para discriminar letras; (b) $22.9 \%$ fue incapaz de dibujar figuras geométricas; (c) aún cuando todos los niños pudieron dibujar la figura humana, 33\% de ellos lo hizo de forma demasiado simple; (d) 36.8\% no fue capaz de identificar los colores primarios, (e) $89.5 \%$ tuvo dificultad para nombrar las figuras geométricas básicas, (f) 72.3\% no pudo repetir series de dígitos, pero el $97.9 \%$ pudo repetir frases con significado correctamente. Habilidades indispensables para acceder a la lecto-escritura y que en general, se aprenden en el nivel preescolar, lo cual se confirma con la correlación significativa obtenida entre la asistencia al preescolar y el desempeño en tareas de integración visomotora e identificación de las figuras geométricas.

Un aspecto evidente es la escasa asistencia de los niños al preescolar; en los dos centros preescolares de la localidad de estudio, la suma de la matrícula escolar en el ciclo 1997-98 fue sólo de 53 niños. Algunas de las razones que las madres otomíes señalan para no enviar a sus hijos al preescolar se reflejan en la siguiente frase: "ya pagamos por los demás niños en la primaria y no podemos pagar para mandar a los más chicos a esta escuela y, además, para que únicamente jueguen". Si atendemos a lo señalado por la Organización Mundial de la Salud con relación a que el 10\% de una población presenta requerimientos de educación especial, es claro que los altos porcentajes de dificultad mostrados constituyen un punto de reflexión y de atención en estas poblaciones.

En cuanto a la variable familia-entorno, datos relevantes indican que los ingresos familiares determinan el nivel educacional de los hijos, pues afectan la capacidad de costear su educación, así como la participación que pudieran tener en la misma; enviarlos a la escuela 
implica gastos directos. Otros aspectos, se vinculan con las aspiraciones educacionales; la mayor parte de los padres otomíes entrevistados no perciben las ventajas de brindarles una mayor educación a los hijos, más allá de saber leer, escribir y contar, lo que les va a asegurar un trabajo en un futuro, que en muchos de los casos no es tan remoto como sucede en el medio urbano, pues hay padres que sacan de la escuela a los niños en el tercer grado de primaria y los "alquilan" para trabajar. Roque (2001) señala que, la falta de apoyo familiar es causada por carecerse de tiempo; tener muchos hijos, además de ocupaciones varias, muchas de las cuales se realizan fuera de la comunidad; ser analfabetas, desconocer la lengua que se prioriza en la enseñanza; o bien, pensar que la educación de los niños compete sólo al maestro; o sentirse desmotivado porque su participación sólo se solicita para actividades ajenas al aprendizaje del menor tales como pintar, barrer, levantar aulas o preparar los desayunos calientes.

Es importante anotar que, en la educación brindada en casa se encuentra aún presente la diferencia por género, aún cuando algunas tareas son realizadas tanto por niñas como por niños. Lo que es un hecho, es que todos, desde muy pequeños, llevan a cabo diferentes actividades; las niñas se ocupan fundamentalmente de quehaceres domésticos en la casa, mientras que los niños realizan actividades fuera de ella. En cuanto al lenguaje, las madres reportaron enseñar a sus hijos a hablar el español más que el otomí, "éste no tiene valor". No obstante, el otomí es hablado tanto en el interior como en el exterior del hogar, de modo que los niños acaban aprendiéndolo, lo entienden porque se lo oyen a sus padres quienes lo utilizan para comunicarse con sus propios padres o con otros adultos. Asimismo, las madres señalaron que no hablaban con sus hijos acerca de cómo iban en la escuela porque, según ellas, "los niños todavía no están en la edad de pensar".

De esta manera se observa que, en la relación maestros-padres, existe también disparidad en la concepción del aprendizaje y en el desarrollo de las habilidades que los niños requieren para funcionar adecuadamente en ambos ambientes. En este sentido, Sandoval (1992) reconoce que la formación del magisterio ha resultado ser, en muchos casos, incapaz de preparar al maestro para enfrentar los diversos problemas que se le presentan cotidianamente en el aula. Esto puede llegar a ser más difícil, cuando el docente es enviado a lugares lejanos y con poblaciones cuya cultura es diferente a la suya. Heshusius (1991) indica que las desigualdades étnicas entre maestros y alumnos puede ser, de entre otras, una de las razones del no 
aprendizaje de los estudiantes, la diferencia cultural puede llevar al maestro a no identificarse con la escuela en la que labora, aspecto que, en algunos casos, fue observado en la escuela.

Lo anteriormente expuesto tiene una repercusión importante en el nivel de aprovechamiento escolar de los menores así como en los índices de deserción y/o permanencia en la escuela. En la relación educación informal-escolarización, hay una clara diferencia. La educación informal brindada en casa conduce al niño al aprendizaje del vivir que se da en cuatro sentidos: (a) su inserción temprana en la organización y economía de la familia; (b) el bilingüismo, (c) las diferencias por cuestiones de género; $y$, (d) el aprendizaje cultural de las expectativas para la vida. Las expectativas escolares de la familia otomí están delimitadas por la cultura, la organización social y la economía campesina. Las diferencias de género son manifiestas desde edades tempranas; los niños colaboran en el mantenimiento del hogar al mismo tiempo que asisten a la escuela. Importante es señalar que estas expectativas difieren de las que maestros, directivos y administradores escolares se plantean, lo que conduce a pensar, que, ambas culturas en este espacio van por vías paralelas (Acle, 2003).

Las diferencias de actitud de los directores escolares y docentes tienen que ver con dos elementos que se entrelazan de manera importante: (1) su propia visión de la educación de los grupos indígenas que, en términos generales, corresponde a la visión de la cultura dominante, por ejemplo: los niños deben dejar de hablar su lengua, deben dejar de vestirse de manera tradicional, la escuela es un espacio de la Secretaría de Educación Pública (SEP) y no de la comunidad, etc.; (2) el tiempo de permanencia en la escuela, mientras más tiempo ocupen el cargo es posible que ello les permitirá conocer y relacionarse más adecuadamente con la comunidad. Ello se logra en pocos casos, pues, la movilidad de los docentes es alta.

Lo anteriormente señalado muestra las redes ecológicas que se establecen en este hábitat creado que es la escuela, al utilizar un enfoque ecológico para detectar los requerimientos educativos especiales de niños de zonas rurales e indígenas, e intervenir en consecuencia, muestra la necesidad de incluir otras variables de análisis, que en su interacción con el menor permitan una comprehensión mayor de este objeto particular de estudio y lleven a propuestas de intervención acordes a esa realidad. Es claro que la tarea no es fácil, pero tampoco se puede seguir centrando en el niño la responsabilidad y mantenimiento de este tipo de dificultades, 
en particular, en poblaciones indígenas, en las que la inadecuada aplicación e interpretación de tests, puede añadir un elemento más de marginación: el de la discapacidad.

\section{Referencias}

Acle, T.G. (2000). Gente de razón: Educación y cultura en Temoaya. Disertación doctoral no publicada. Universidad Iberoamericana, Distrito Federal, México.

Acle, T.G. (2003). Ecología de la Educación Especial. Revista Nueva Antropología, 19, 62, 29-53.

Acle, T. G. y Roque, H. M. P. (1994). Necesidades de Educación Especial en una Población Otomí del Estado de México. La Psicología Social en México, 5, 486-492.

Acle T.G., Roque H. M.P. y Contreras, R.E.A. (1996). Detección de problemas de aprendizaje en niños otomíes de primer grado: Un enfoque ecológico. La Psicología Social en México, 6, 381-386.

Aguirre, G. (1992). El proceso de aculturación y el cambio sociocultural en México. Obra Antropológica VI. México: Fondo de Cultura Económica.

Báez de la Fé, B. F. (1989). Del Estudio de Caso al Análisis de la Situación: Evolución Histórica del Diagnóstico Psicoeducativo. Infancia y Aprendizaje, 46, 71-81.

Heshusius, L. (1991). Future Perspectives. En K. D. Reid, W. P. Hresko y H. L. Swanson, (Eds.), A Cognitive Approach to Learning Disabilities. Texas, EE. UU. : PRO-ED.

Izquierdo, M., Fernández, L. y Nieto, J., (1992). Modelo de educación especial para el medio indígena. Mecanograma. México.

Kirk, S.A.y Gallagher, J., (1989). Educating Exceptional Children. EE. UU.: Houghton Mifflin Co.

Paéz, G.R. y Trejo, A. J., (1994). Detección de niños con requerimientos de educación especial en áreas rurales. La Psicología Social en México. 5, 480-485.

Roque, H. M.P. (2001). Estudio de la participación parental en una escuela monolingüe español de una comunidad otomi desde un enfoque ecológico. Tesis de maestría no publicada, Facultad de Estudios Superiores Zaragoza. U.N.A.M; México.

Rosenberg, M. (1987). Beyond Finding and Fixing the Deficiencies: Augmenting the Clinical Model. Journal of Reading, Writing, and Learning Disabilities International, 3, 291296. 
Sandoval, E. (1992). Relaciones y saberes docentes en los cursos de actualización. En E. Rockwell; La escuela cotidiana. (pp. 88-119). México: Fondo de Cultura Económica.

Serrano, C.E., Embriz, O.A., y Fernández, H.P. (2002). Indicadores socioeconómicos de los pueblos indígenas de México, 2002. México: I.N.I.-U.N.D.P.-CONAPO.

Sugai, G. y Maheady, L. (1988). Cultural Diversity and Individual Assessment for Behavior Disorders. Teaching Exceptional Children, Otoño, 28-31.

Swartz, J.L. \& Martín, W. (1997) Applied ecological psychology for schools within communities. Ney Jersey, EE.UU.: Erlbaum. 